\title{
OPEN Distinct DNA methylation patterns associated with treatment resistance in metastatic castration resistant prostate cancer
}

\author{
Madonna R. Peter ${ }^{1,2}$, Misha Bilenky ${ }^{3}$, Alastair Davies ${ }^{4}$, Ruth Isserlin ${ }^{5}$, Gary D. Bader ${ }^{5}$, \\ Neil E. Fleshner ${ }^{6}$, Martin Hirst ${ }^{3,7}$, Amina Zoubeidi $^{4}$ \& Bharati Bapat ${ }^{1,2 凶}$
}

Androgens are a major driver of prostate cancer ( $\mathrm{PCa}$ ) and continue to be a critical treatment target for advanced disease, which includes castration therapy and antiandrogens. However, resistance to these therapies leading to metastatic castration-resistant prostate cancer (mCRPC), and the emergence of treatment-induced neuroendocrine disease (tNEPC) remains an ongoing challenge. Instability of the DNA methylome is well established as a major hallmark of PCa development and progression. Therefore, investigating the dynamics of the methylation changes going from the castration sensitive to the tNEPC state would provide insights into novel mechanisms of resistance. Using an established xenograft model of CRPC, genome-wide methylation analysis was performed on cell lines representing various stages of PCa progression. We confirmed extensive methylation changes with the development of CRPC and tNEPC using this model. This included key genes and pathways associated with cellular differentiation and neurodevelopment. Combined analysis of methylation and gene expression changes further highlighted genes that could potentially serve as therapeutic targets. Furthermore, tNEPC-related methylation signals from this model were detectable in circulating cell free DNA (cfDNA) from mCRPC patients undergoing androgen-targeting therapies and were associated with a faster time to clinical progression. These potential biomarkers could help with identifying patients with aggressive disease.

Prostate Cancer $(\mathrm{PCa})$ is an androgen driven disease, with androgen-deprivation therapy (ADT) remaining the most predominant treatment for metastatic $\mathrm{PCa}^{1}$. Although initially beneficial, resistance to ADT is inevitable, leading to metastatic castration resistant prostate cancer (mCRPC) ${ }^{2,3}$. These tumors often continue to rely on the androgen pathway despite castrate/low levels of androgens through various mechanisms, including mutations in the androgen receptor $(A R)$ gene and extragonadal androgen production ${ }^{4}$. As a result, the treatment landscape for mCRPC includes therapies that target extragonadal androgen biosynthesis (abiraterone acetate) and AR activation (i.e. enzalutamide) $)^{5-8}$. Much focus in recent clinical trials is determining optimal therapy sequences at various stages of PCa, from the castration-naïve setting to the mCRPC state ${ }^{9,10}$. While all of these treatments have been shown to improve survival, resistance occurs through various AR driven mechanisms and alternative lineage re-programming, owing to the molecular heterogeneity of $\mathrm{PCa}$, which could lead to treatment induced neuroendocrine prostate cancer $(\mathrm{tNEPC})^{11-13}$. Tracking the molecular changes that occur with each treatment could facilitate personalized treatment decisions ${ }^{14}$.

Extensive genomic analysis of mCRPC tumors has revealed molecular aberrations beyond the $A R$ gene, implicating several oncogenic pathways, such as cell cycle regulation, apoptosis, and DNA repair ${ }^{11,15}$. In addition, epigenomic changes that occur with the development of PCa and during treatment are also a major contributor to the phenotypic heterogeneity among patients ${ }^{16}$. These alterations include chromatin remodeling, transcriptomic

\footnotetext{
${ }^{1}$ Lunenfeld-Tanenbaum Research Institute, Sinai Health System, 60 Murray Street, Toronto, ON M5T 3L9, Canada. ${ }^{2}$ Department of Laboratory Medicine and Pathobiology, University of Toronto, Toronto, Canada. ${ }^{3}$ Canada's Michael Smith Genome Science Center, BC Cancer Agency, Vancouver, Canada. ${ }^{4}$ Vancouver Prostate Centre, Vancouver, BC, Canada. ${ }^{5}$ Terrence Donnelly Centre for Cellular and Biomolecular Research, University of Toronto, Toronto, Canada. ${ }^{6}$ Division of Urology, Departments of Surgery and Surgical Oncology, University Health Network, Toronto, Canada. ${ }^{7}$ Department of Microbiology and Immunology and Michael Smith Laboratories, University of British Columbia, Vancouver, Canada. ${ }^{\circledR}$ email: bapat@lunenfeld.ca
} 
a)

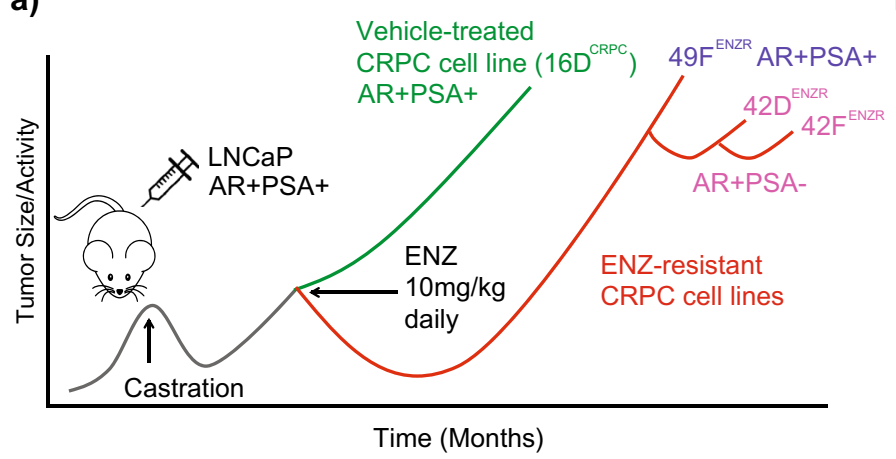

c)

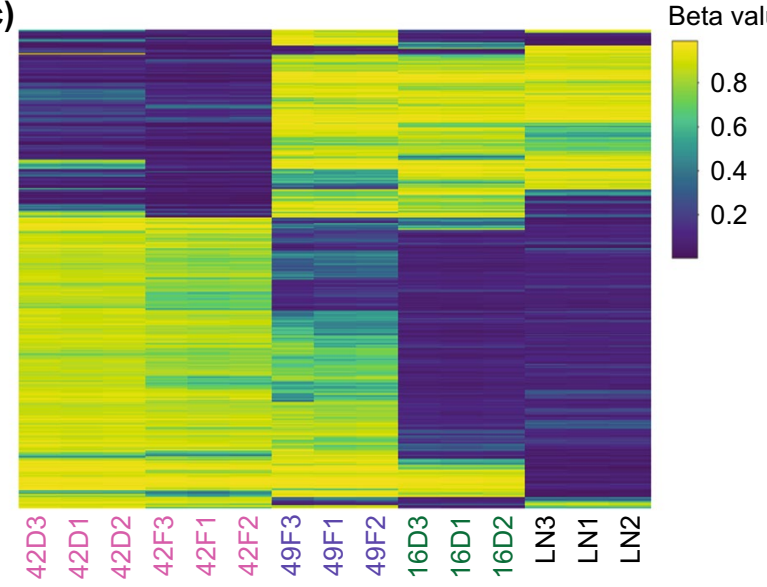

b)

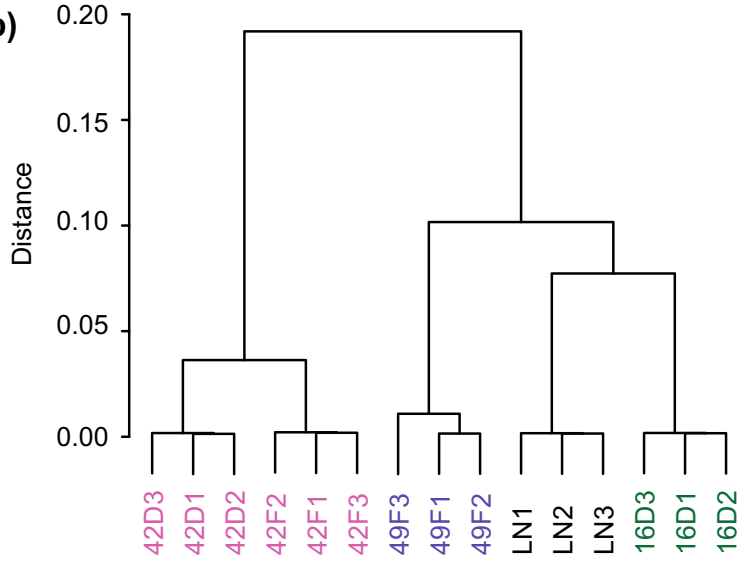

Figure 1. CRPC xenograft model and DNA methylation patterns across all cell lines. (a) Previously, LNCaP (LN) cells were injected into nude mice and allowed to grow into tumors. Following castration, CRPC-like cells/ tumors emerge. The mice were either treated with vehicle-control or enzalutamide to create control CRPC cells $\left(16 \mathrm{D}^{\mathrm{CRPC}}\right)$ or treatment resistant cells $\left(49 \mathrm{~F}^{\mathrm{ENZR}} / 42 \mathrm{D}^{\mathrm{ENZR}} / 42 \mathrm{~F}^{\mathrm{ENZR}}\right)$, respectively. (b) Unsupervised hierarchical clustering of all detectable methylation array probes of each cell line (in triplicate) using the Ward linkage method. (c) Heatmap of the methylation levels (beta values) of the top 1000 differentially methylated probes (DMPs). (d) Commonly altered DMPs related to emergence of CRPC was found by comparing all CRPC cell lines to LN. Common enzalutamide resistance (ENZR) associated DMPs were determined through comparison with control CRPC ells $\left(16 \mathrm{D}^{\mathrm{CRPC}}\right)$ and $\mathrm{NNEPC}$ related changes by comparing with $49 \mathrm{~F}^{\mathrm{ENZR}}$ cells.

changes modulated by non-coding RNA, and altered DNA methylation ${ }^{17-19}$. In mCRPC, DNA methylation patterns were better able to distinguish Adenocarcinoma-CRPC (CRPC-Adeno) from a highly aggressive form of the disease, Neuroendocrine-CRPC (CRPC-NE) ${ }^{20}$, which is characterized by low canonical AR activity, frequent loss of tumor suppressor genes (i.e. RB1 and TP53), concomitant with expression of neuronal lineage markers (i.e. chromogranin A and neuron-specific enolase) as well as stem-cell like factors (i.e. CD44) ${ }^{21}$. While the molecular drivers of trans-differentiation from CRPC-Adeno to CRPC-NE are still emerging, epigenomic changes likely play a significant role ${ }^{22}$. For instance, DNA methyltransferase 1 (DNMT1) is highly expressed in CRPC-NE tumors compared to CRPC-Adeno ${ }^{20,23}$.

In order to investigate the epigenomic mechanisms that drive CRPC-NE development, we assessed methylation alterations during distinct stages of prostate cancer progression. Using cell lines derived from a xenograft model, we analyzed the DNA methylome during the transition from a hormone/castration-sensitive state to CRPC to enzalutamide-resistant (ENZR) CRPC, including both CRPC-Adeno and CRPC-NE phenotypes. In particular, we distinguished methylation patterns associated with the development of CRPC-Adeno from CRPCNE, highlighting key mechanisms and potential therapeutic targets specific to these clinical states. In addition, we integrated our findings from this pre-clinical model with circulating cell free DNA (cfDNA) from mCRPC patients to identify potential biomarkers associated with aggressive disease.

\section{Results}

Changes in DNA methylation patterns associated with the development of CRPC and enzalutamide resistance. Previously, CRPC and enzalutamide resistant (ENZR) CRPC cell lines were derived from a LNCaP xenograft mouse model (Fig. 1a ${ }^{24,25}$. In this study, the methylome of the castration sensitive LNCaP

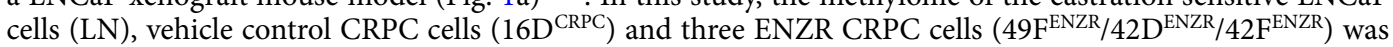
assessed. Mimicking the clinical diversity of enzalutamide resistance, both AR-driven CRPC-Adeno (49F ${ }^{\mathrm{ENZR}}$ ) and CRPC-NE/tNEPC $\left(42 \mathrm{D}^{\mathrm{ENZR}}, 42 \mathrm{~F}^{\mathrm{ENZR}}\right)$ phenotypes were examined. We performed methylation array profiling of $>485,000$ probes/CpG sites across the genome. As expected, unsupervised clustering analysis of methyla- 
tion levels across all probes show close clustering of replicates, with distinct methylation patterns among ENZR cells compared to LN and $16 \mathrm{D}^{\mathrm{CRPC}}$ cells (Fig. 1b, c). The tNEPC cells, $42 \mathrm{D}^{\mathrm{ENZR}}$ and $42 \mathrm{~F}^{\mathrm{ENZR}}$, demonstrated the most divergent methylation signals from LN cells.

Increased differentially methylated probes/CpG sites with the development of ENZR and tNEPC. We next performed differential methylation analysis to identify changes associated with: (a) the transition from castration sensitive to the castration resistant state (common changes between all CRPC/ENZR cells vs LN), (b) the development of enzalutamide resistance (all ENZR cells vs $16 \mathrm{D}^{\mathrm{CRPC}}$ ) and finally (c) the tNEPC state $\left(42 \mathrm{D}^{\mathrm{ENZR}} / 42 \mathrm{~F}^{\mathrm{ENZR}}\right.$ vs $\left.49 \mathrm{~F}^{\mathrm{ENZR}}\right)$ (Fig. $\left.1 \mathrm{~d}\right)$. We applied $\mathrm{FDR}(<0.01)$ and $\log \mathrm{FC}(\geq 0.2$, fold-change) cut-offs to determine differentially methylated probes (DMPs) (Supplementary Tables 1-9). With the development of enzalutamide resistance ( $\left.49 \mathrm{~F}^{\mathrm{ENZR}} / 42 \mathrm{D}^{\mathrm{ENZR}} / 42 \mathrm{~F}^{\mathrm{ENZR}} \mathrm{vs} \mathrm{LN}\right)$, there was a larger number of DMPs compared to vehicle control cells ( $16 \mathrm{D}^{\mathrm{CRPC}}$ vs LN), especially hypermethylated CpGs (Fig. 2a). Overall, all comparisons showed a similar $\log$ FC distribution, and were not significantly different between comparisons (Fig. 2b). The tNEPC cell lines, $42 \mathrm{D}^{\mathrm{ENZR}} / 42 \mathrm{~F}^{\mathrm{ENZR}}$, tended to have the most DMPs (vs LN and vs $16 \mathrm{D}^{\mathrm{CRPC}}$ ). The overall distribution of DMPs within transcriptional start sites/promoters (TSS, 0-1500 bp upstream), untranslated regions (UTRs), gene bodies and intergenic regions (IGR) did not vary across comparisons (Supplementary Figure 1a). Similar proportions of DMPs within CpG islands (CGIs), shores, shelves and open sea regions were also found across cell line comparisons (Supplementary Figure 1b). The proportion of hypermethylated versus hypomethylated DMPs for each genomic region confirmed tendency towards increased methylation of CpG sites analyzed in ENZR CRPC cells compared to parental (LN) or control CRPC (16D ${ }^{\mathrm{CRPC}}$ ) cells (Supplementary Figure 1c-f).

To find critical methylation changes associated with the development of CRPC, we examined all cell lines vs LN comparisons, including $16 \mathrm{D}^{\mathrm{CRPC}}$ vs LN and ENZR cells vs LN. This helped to refine key changes specific to overall development of CRPC. Similarly, common DMPs associated with enzalutamide resistance $\left(49 \mathrm{~F}^{\mathrm{ENZR}} / 42 \mathrm{D}^{\mathrm{ENZR}} / 42 \mathrm{~F}^{\mathrm{ENZR}} \mathrm{vs} 16 \mathrm{D}^{\mathrm{CRPC}}\right)$, and the neuroendocrine phenotype $\left(42 \mathrm{D}^{\mathrm{ENZR}} / 42 \mathrm{~F}^{\mathrm{ENZR}} \mathrm{vs}^{\mathrm{E}} 4 \mathrm{~F}^{\mathrm{ENZR}}\right)$ was also examined. With the development of CRPC and enzalutamide resistant states, the majority of common DMPs (vs LN or vs $16 \mathrm{D}^{\mathrm{CRPC}}$ ) were hypermethylated (Fig. 2c). In contrast, there were nearly equal numbers of hypermethylated (10,600 DMPs) and hypomethylated (10,722 DMPs) DMPs commonly found between tNEPC cells compared to the CRPC-Adeno cells $\left(42 \mathrm{D}^{\mathrm{ENZR}} / 42 \mathrm{~F}^{\mathrm{ENZR}}\right.$ vs $\left.49 \mathrm{~F}^{\mathrm{ENZR}}\right)$. Correlation analysis of common DMPs showed significant concordance of these shared DMPs across vs LN, vs $16 \mathrm{D}^{\mathrm{CRPC}}$ and vs $49 \mathrm{~F}^{\mathrm{ENZR}}$ comparisons (Supplementary Figure 2). We further stratified these common DMPs by genomic location, with TSS/promoters, gene bodies and intergenic regions being the most represented (Fig. 2d).

We also examined whether there were any probes that overlapped going from the castration-sensitive to CRPC state and finally to the ENZR CRPC phenotype. There were 507 DMP sites associated with both CRPC development and enzalutamide resistance, the majority shared the same trend (i.e. 384 hypermethylated probes vs LN and vs $16 \mathrm{D}^{\mathrm{CRPC}}$ ) and few changed directionality (i.e. 29 probes hypomethylated vs LN and hypermethylated in vs $16 \mathrm{D}^{\mathrm{CRPC}}$ comparisons) (Fig. 2e). Interestingly, 107 probes were hypermethylated with the development of ENZR (vs $16 \mathrm{D}^{\mathrm{CRPC}}$ ) but were hypomethylated in $42 \mathrm{D}^{\mathrm{ENZR}} / 42 \mathrm{~F}^{\mathrm{ENZR}}$ cells compared to $49 \mathrm{~F}^{\mathrm{ENZR}}$ (Fig. $2 \mathrm{f}$ ), suggesting that reduced methylation in $42 \mathrm{D}^{\mathrm{ENZR}} / 42 \mathrm{~F}^{\mathrm{ENZR}}$ of these DMPs may be important for the transition to the tNEPC-like state. Indeed, global hypomethylation is associated with more genomic instability and found to be associated with PCa progression ${ }^{26}$. While 20/107 CpGs are located in intergenic regions, the remaining were found within/ near several genes (Supplementary Table 10). We next examined the pathways and genes associated with ENZR and $\mathrm{tNEPC}$ development.

Pathways and genes associated with CRPC development and enzalutamide resistance. We performed pathway analysis of all DMPs (Supplementary Tables 11-19) and examined pathways shared by all CRPC cells vs LN, ENZR cells vs $16 \mathrm{D}^{\mathrm{CRPC}}$, and tNPEC cells vs $49 \mathrm{~F}^{\mathrm{ENZR}}$ comparisons. Interestingly, there were very few pathways with methylation changes specific to the development of CRPC (vs LN) alone, with the majority (204) shared across comparisons (Fig. 3a). Common pathways with the development of the CRPC state includes those involved in developmental processes, cellular differentiation, MAP kinase signaling and ion homeostasis (Fig. 3b). When we examined ENZR related pathways $\left(49 \mathrm{~F}^{\mathrm{ENZR}} / 42 \mathrm{D}^{\mathrm{ENZR}} / 42 \mathrm{~F}^{\mathrm{ENZR}}\right.$ vs $\left.16 \mathrm{D}^{\mathrm{CRPC}}\right)$, there were several hormone transport/secretion pathways implicated as well as immunomodulatory pathways, including leukocyte migration and lymphocyte activation. ENZR tNEPC cells compared to CRPC-Adeno cells tended to exhibit more embryonic, morphogenic and neuronal development pathways (Fig. 3b and Supplementary Tables 18-19). We next focused on genes associated with these DMPs, particularly near or within protein coding genes. Overall, we found 2575 genes with differentially methylated CpG sites (TSS/promoters and gene bodies combined) shared between all comparisons, and additional genes associated with the development of enzalutamide resistance and the tNEPC phenotype (Fig. 3c). However, as the array only samples a fraction of the $\sim 28$ million CpG sites in the genome, certain genes are better represented in terms of number of CpGs analyzed than others, with an average of $47 \%$ (range 30-60\%) of genes with only 1-2 DMPs. In order to further refine important genes/ pathways associated with disease progression, we integrated cell line methylation data with published data sets.

In a recent whole genome bisulfite sequencing study (WGBS) of mCRPC tumors, distinctive methylation patterns were observed amongst patients, including those patients with neuroendocrine disease ${ }^{27}$. We assessed the overlap of these methylated regions with DMPs from ENZR vs $16 \mathrm{D}^{\mathrm{CRPC}}$ and $\mathrm{tNEPC}$ vs $49 \mathrm{~F}^{\mathrm{ENZR}}$ comparisons and further refined 209 genes with more than 5 DMPs. We calculated the net methylation change by scoring the ratio of hypermethylated to hypomethylated probes within each gene (Supplementary Figure 3). There were 81 genes that were hypermethylated with the development of ENZR and further hypermethylated among tNEPC cells. These genes included those involved in regulation of cell cycle $(C R E B 5, E R G)^{28,29}$, tumor suppressors $(C T N N A 2, O P C M L)^{30,31}$, as well as genes that regulate neurodevelopment (AUTS2, SYNGAP1) ${ }^{32,33}$. Furthermore, 
a)

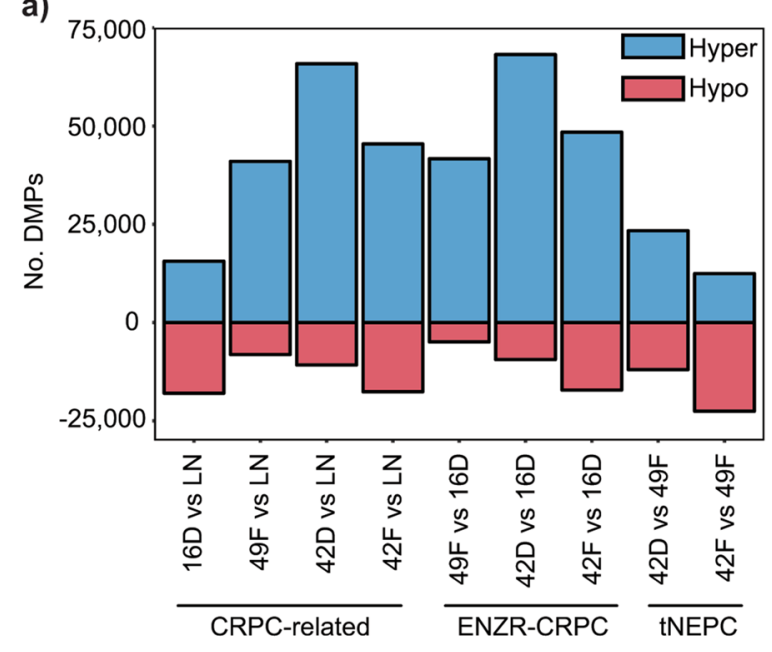

b)

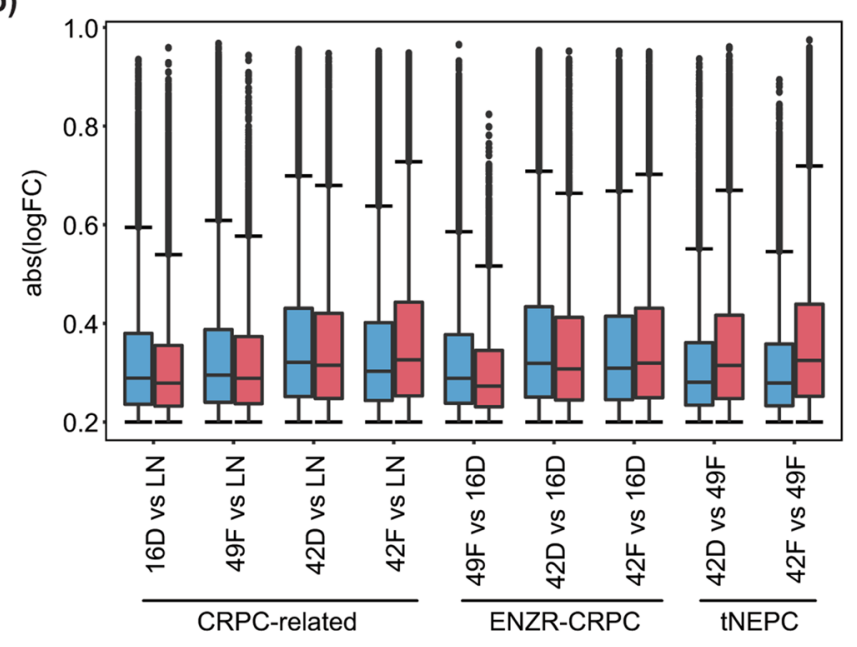

c)

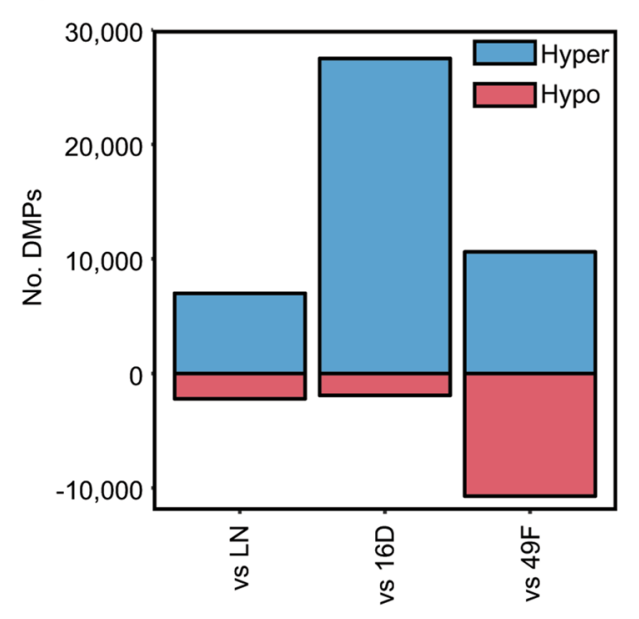

e)

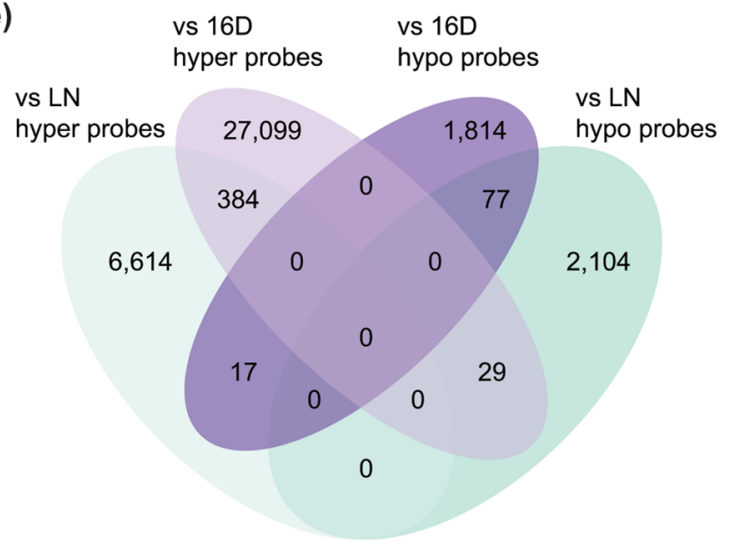

d)

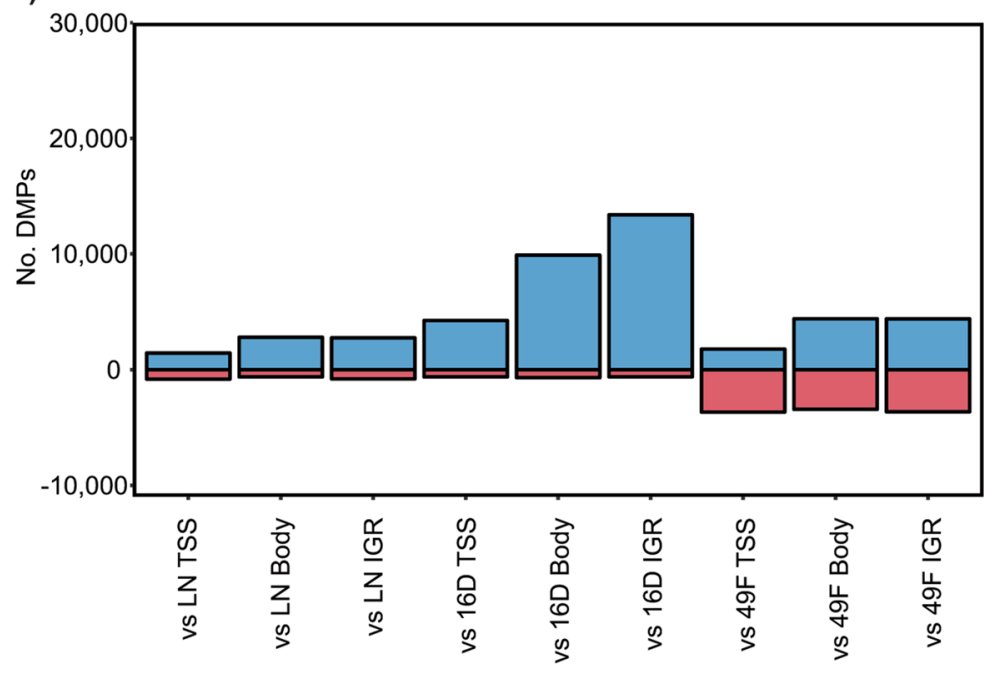

f)

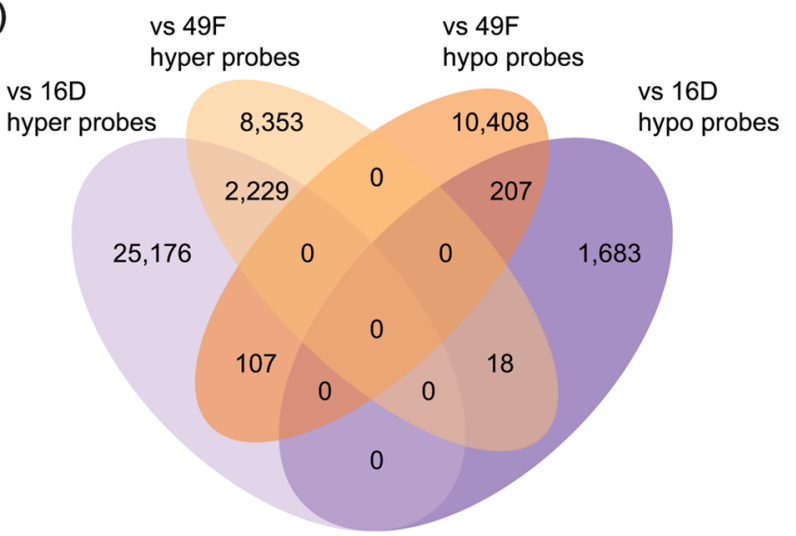

Figure 2. Overview of differentially methylated probes for all cell comparisons. (a) The total number of hypermethylated and hypomethylated probes for all comparisons is shown (b) Box plot summarizes the distribution of the absolute logFC values for all DMPs within each comparison. (c) Summary of the number of hypermethylated and hypomethylated DMPs shared by all vs LN, or vs $16 \mathrm{D}^{\mathrm{CRPC}}$ or vs $49 \mathrm{~F}^{\mathrm{ENZR}}$ comparisons. (d) For TSS regions, gene bodies and intergenic regions, the total number of hypermethylated and hypomethylated DMPs is shown. (e) Venn diagram illustrates the extent of overlap between vs LN and vs $16 \mathrm{D}^{\mathrm{CRPC}} \mathrm{DMPs}$ according to methylation trend. (f) Similarly, the shared DMPs between vs $49 \mathrm{~F}^{\mathrm{ENZR}}$ and vs $16 \mathrm{D}^{\mathrm{CRPC}}$ comparisons are illustrated. 
a)

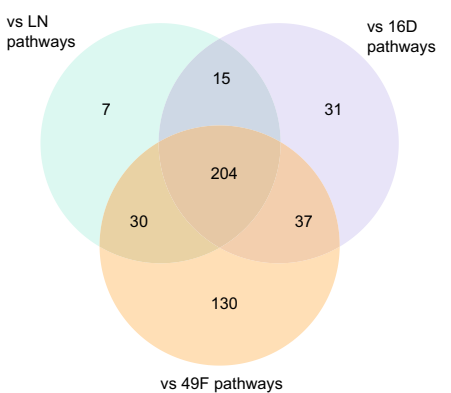
vs LN genes

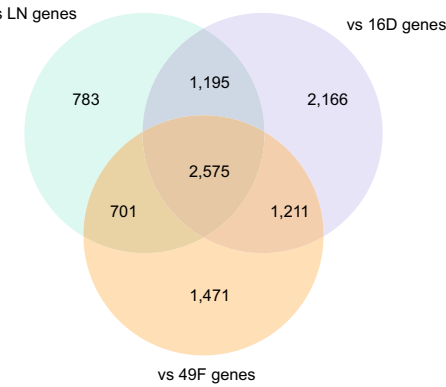

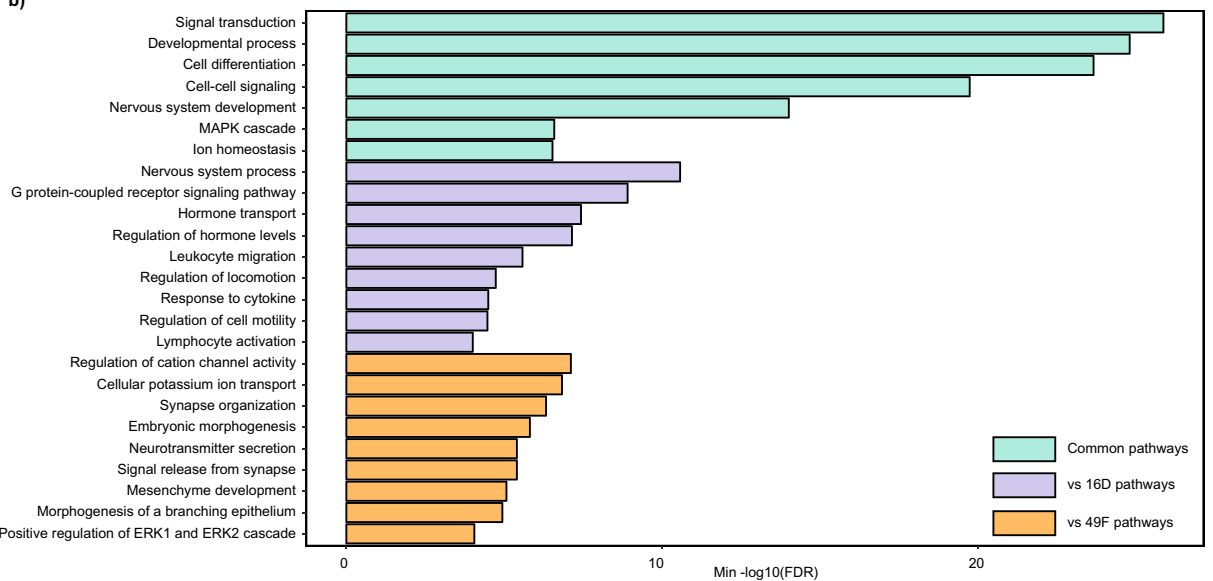
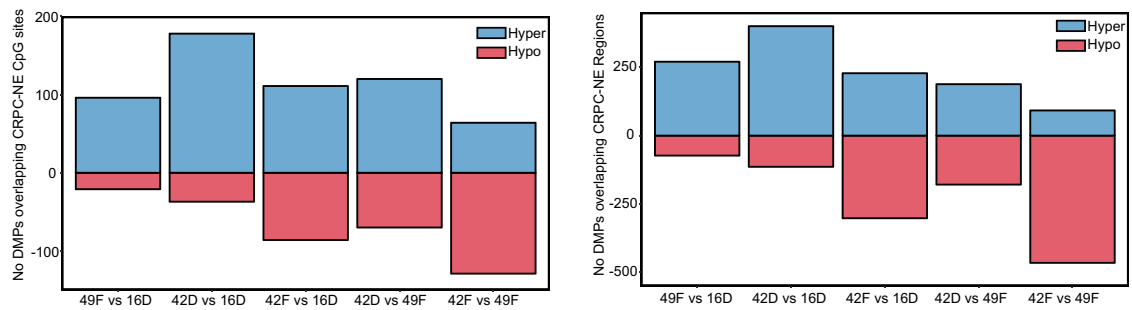

Figure 3. Summary of common pathways and comparison with tNEPC tumor-derived methylation patterns. (a) Venn diagram highlighting the common pathways associated with the development of CRPC, enzalutamide resistance and with the emergence of the tNEPC state. (b) Representative pathways with differentially methylated genes for all comparisons and ranked by the minimum - $\log 10$ (FDR) values. (c) Venn diagram illustrates the overlap in the number of genes with DMPs between vs LN, vs $16 \mathrm{D}^{\mathrm{CRPC}}$ and vs $49 \mathrm{~F}^{\mathrm{ENZR}}$ comparisons (TSS and gene bodies combined). (d) The DMPs from vs $16 \mathrm{D}^{\mathrm{CRPC}}$ and vs $49 \mathrm{~F}^{\mathrm{ENZR}}$ comparisons were integrated with CRPC-NE vs CRPC-Adeno DMCs from a biopsy tissue study. The number of hypermethylated and hypomethylated DMPs that demonstrated the same methylation trends as CRPC-NE tumors is represented in the bar plot. (e) Similarly, DMPs were overlaid with known CRPC-NE regions previously detected in cfDNA and quantified for all ENZR cell lines.

there were 19 genes that were hypermethylated in $49 \mathrm{~F}^{\mathrm{ENZR}}$ vs $16 \mathrm{D}^{\mathrm{CRPC}}$, but hypomethylated in $42 \mathrm{D}^{\mathrm{ENZR}} / 42 \mathrm{~F}^{\mathrm{ENZR}}$ cells, including the $A R$ gene and development related genes (PITX2, ROBO1/2) ${ }^{34,35}$.

As several neurodevelopmental genes were implicated in our analysis, we wanted to examine whether these DMPs are also found within $\mathrm{TNEPC}$ tumor tissue. In a prior study analyzing CRPC-NE vs CRPC-Adeno biopsy tissue, differentially methylated CpG sites (DMCs) between these disease states were identified ${ }^{20}$. In a follow-up study, these CRPC-NE/tNEPC associated methylation patterns could be detected in matched cfDNA samples ${ }^{36}$. We first compared biopsy-derived DMCs with DMPs and found that $\mathrm{tNEPC}$ cells $\left(42 \mathrm{D}^{\mathrm{ENZR}} / 42 \mathrm{~F}^{\mathrm{ENZR}}\right)$ tended to have more DMPs that overlapped with CRPC-NE tissue than $49 \mathrm{~F}^{\mathrm{ENZR}}$ (Fig. 3d). However, this biopsy study utilized reduced representation bisulfite sequencing (RRBS), which would have CpG sites not represented in the array in this study. We also examined whether vs $16 \mathrm{D}^{\mathrm{CRPC}}$ and vs $49 \mathrm{~F}^{\mathrm{ENZR}}$ DMPs were detectable in cfDNA samples from confirmed CRPC-NE patients ${ }^{36}$, and found a similar pattern with more overlapping DMPs from the tNEPC cell lines (Fig. 3e). There were some CRPC-NE associated methylation patterns in $49 \mathrm{~F}^{\mathrm{ENZR}}$ cells, but not to the same extent as $42 \mathrm{D}^{\mathrm{ENZR}} / 42 \mathrm{~F}^{\mathrm{ENZR}}$ cells.

Promoter versus gene body methylation and impact on gene expression. DNA methylation alterations in promoters and gene bodies are known to impact gene expression ${ }^{37}$. For instance, hypermethylation in promoter/TSS regions can be associated with suppression of gene expression, whereas hypermethylation in gene body regions could be associated with increased expression. Among genes with DMPs in TSS regions ( $\geq 3$ DMPs) and body regions ( $\geq 5$ DMPs), the majority of genes were specifically altered in ENZR cell lines (Supplementary Figure $4 \mathrm{a}-\mathrm{b}$ ). In order to further investigate the impact of the DNA methylation changes on genes expression, especially with the development of tNEPC-like disease, we integrated DNA methylation data with RNA-seq expression data, which was previously generated for LNCaP, $16 \mathrm{D}^{\mathrm{CRPC}}, 42 \mathrm{D}^{\mathrm{ENZR}}$ and $42 \mathrm{~F}^{\mathrm{ENZR}}$ cell lines ${ }^{38}$. We performed differential expression analysis comparing $16 \mathrm{D}^{\mathrm{CRPC}}$ and ENZR cells to LN as well as $42 \mathrm{D}^{\mathrm{ENZR}} / 42 \mathrm{~F}^{\mathrm{ENZR}}$ versus $16 \mathrm{D}^{\mathrm{CRPC}}$, and identified genes that are upregulated or downregulated for these comparisons (Supplementary Tables 20-24). The overall number of differentially expressed genes tended to increase with the development of tNPEC phenotype (Supplementary Figure 4c), and very few overlapped across all vs LN 
a)

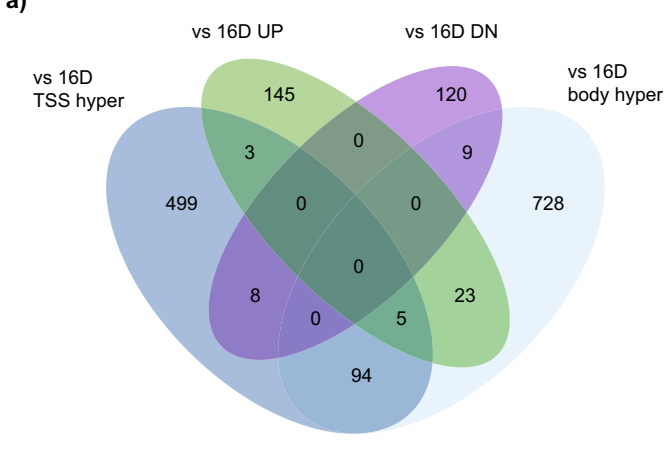

b)

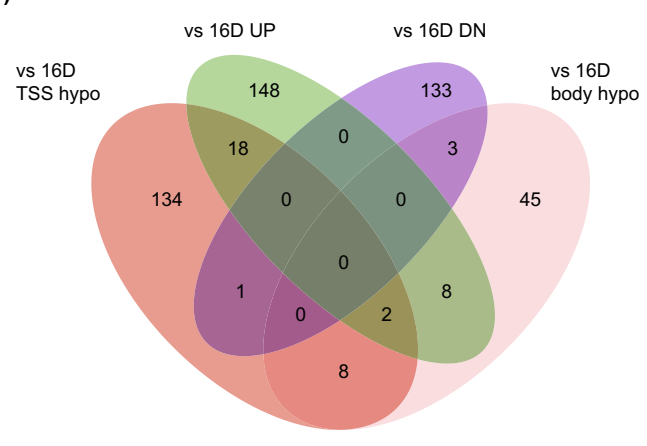

c)

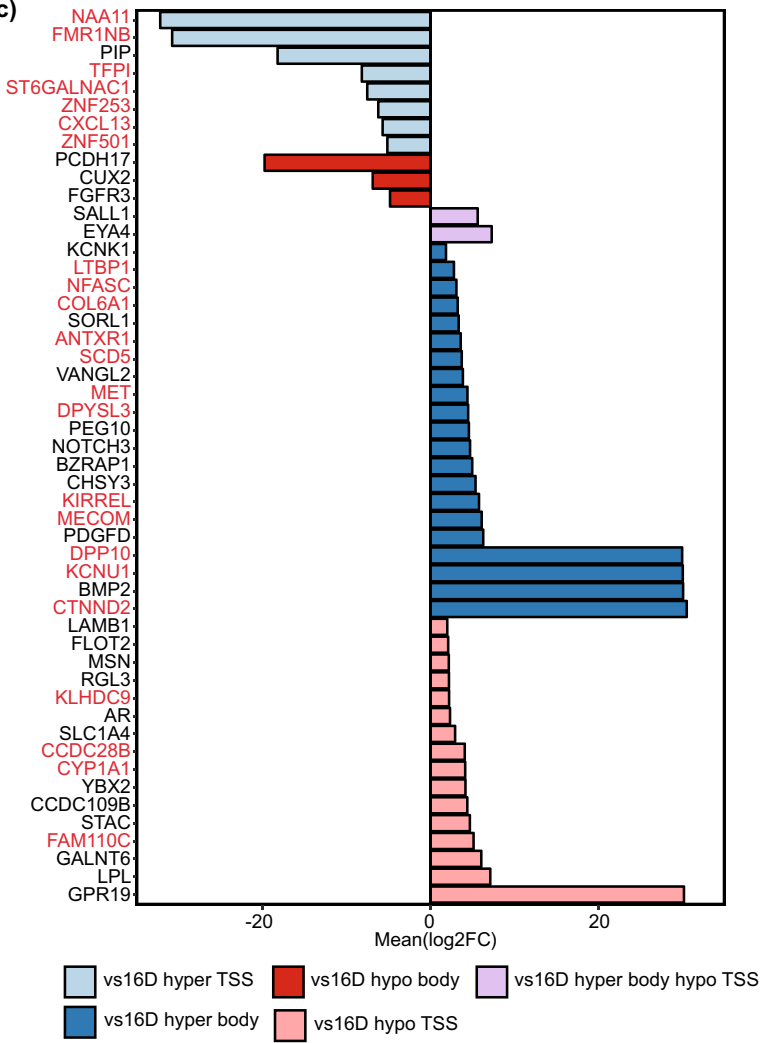

Figure 4. Integrated analysis of enzalutamide resistance related DNA methylation changes with gene expression changes. (a) Venn diagram examines genes that are hypermethylated in tNEPC cells vs $16 \mathrm{D}^{\mathrm{CRPC}}$ TSS regions and/or gene bodies as well as with differential expression ( $\mathrm{UP}=$ upregulated genes in $42 \mathrm{D}^{\mathrm{ENZR}} / 42 \mathrm{~F}^{\mathrm{ENZR}}$, $\mathrm{DN}=$ downregulated genes). (b) Similarly, the extent of overlap between tNPEC cells vs $16 \mathrm{D}^{\mathrm{CRPC}}$ hypomethylated genes is shown. (c) Bar plot shows all genes with differential expression and associated changes in DNA methylation in tNEPC cells. Mean $\log 2 \mathrm{FC}$ for $42 \mathrm{D}^{\mathrm{ENZR}} / 42 \mathrm{~F}^{\mathrm{ENZR}} \mathrm{vs} 16 \mathrm{D}^{\mathrm{CRPC}}$ is shown. Genes with similar methylation patterns in $49 \mathrm{~F}^{\mathrm{ENZR}}$ cells are indicated with pink text.

comparisons, with most genes differentially expressed in $42 \mathrm{D}^{\mathrm{ENZR}} / 42 \mathrm{~F}^{\mathrm{ENZR}}$ cells (Supplementary Figure $4 \mathrm{~d}-\mathrm{g}$ ). We confirmed aberrant expression of AR regulated genes in all CRPC cell lines $\left(16 \mathrm{D}^{\mathrm{CRPC}} / 42 \mathrm{D}^{\mathrm{ENZR}} / 42 \mathrm{~F}^{\mathrm{ENZR}}\right.$ vs LN), including loss of TMPRSS2 expression (Supplementary Figure 5a) ${ }^{39}$. The tNEPC cell lines and not $16 \mathrm{D}^{\mathrm{CRPC}}$ cells demonstrated reduced expression of prostate-specific antigen (PSA/KLK3) compared to LN (Supplementary Tables 20-22). The development of the tNEPC phenotype led to further alterations in known AR pathway regulated genes $\left(42 \mathrm{D}^{\mathrm{ENZR}} / 42 \mathrm{~F}^{\mathrm{ENZR}}\right.$ vs $\left.16 \mathrm{D}^{\mathrm{CRPC}}\right)$, including increased expression of $W N T 5 A$, which could be involved in the progression of aggressive disease ${ }^{40,41}$, and downregulation of a suppressor of metastasis, $N D R G 1^{42}$ (Supplementary Figure 5b).

For all vs LN differentially expressed genes, we assessed the following scenarios: (a) genes that are downregulated with promoter hypermethylation and no gene body hypermethylation, (b) genes that are upregulated with gene body hypermethylation and no promoter hypermethylation, (c) genes that were downregulated with gene body hypomethylation and no promoter hypomethylation, and (d) upregulated genes with promoter hypomethylation and no body hypomethylation (Supplementary Figure 6a-b). Compared to LN cells, there were only 12 genes with DMPs that were commonly downregulated in all CRPC cells (11 with scenario a, XKR6 with scenario c), including the ion transporter SLC22A3, which was shown to be downregulated in a subset of head and neck cancers $^{43}$, and a regulator of neuronal development, GPR126 $6^{44}$ (Supplementary Figure 6c). We next focused on genes specifically altered in tNEPC-like cell lines.

In terms of changes associated with development of neuroendocrine features $\left(42 \mathrm{D}^{\mathrm{ENZR}} / 42 \mathrm{~F}^{\mathrm{ENZR}}\right.$ vs $\left.16 \mathrm{D}^{\mathrm{CRPC}}\right)$, we examined the same scenarios as the LN comparisons (Fig. 4 a, b). All genes with DMPs in either TSS or body regions with accompanying expression changes are shown in Fig. 4c. While RNA expression data for $49 \mathrm{~F}^{\mathrm{ENZR}}$ cells was not available, we highlighted genes that showed similar methylation patterns in both tNPEC and CRPCAdeno cells, which included promoters/markers of stem like phenotype (ST6GALNAC1, ANTXR1) ${ }^{45,46}$, and mediators of cell motility and growth (CXCL13, COL6A1) $)^{47,48}$. Alterations specific to the CRPC-NE phenotype, included mediators of development $(\mathrm{BMP} 2, \mathrm{NOTCH} 3)^{49,50}$, and regulators of epithelial-mesenchymal transition (FLOT2, PCDH17) $)^{51,52}$. Interestingly, $42 \mathrm{D}^{\mathrm{ENZR}} / 42 \mathrm{~F}^{\mathrm{ENZR}}$ cells also exhibited increased expression of PEG10, which was shown to be upregulated in CRPC-NE tumors ${ }^{53}$. In addition, tNEPC cells showed a slight increase in AR expression with hypomethylated TSS DMPs, suggesting that the AR pathway may still be utilized, but in a non-canonical fashion as these cells do not express PSA ${ }^{38}$. Few of these genes with combined methylation and gene expression changes are potentially regulated by the AR, including CTNND2, CUX2 and CXCL13 ${ }^{39}$. In 
particular, increased expression of a Wnt pathway gene, CTNND2, could be involved in resistance to AR targeting therapies ${ }^{54}$.

ENZR-related methylation patterns in circulating DNA from mCRPC patients. Recently, we conducted a study collecting sequential cfDNA samples from mCRPC patients receiving androgen-targeting treatments, including enzalutamide and abiraterone acetate ${ }^{55}$. We tracked cfDNA methylation changes using genome-wide sequencing for each patient starting from prior to initiation of treatment (Visit A), at around 12 weeks during treatment (Visit B), and upon clinical progression (Visit C). When we examined the overlap between differentially methylated regions (DMRs) in cfDNA with CRPC-NE tissue related DMCs ${ }^{20}$, we found that patients that harbored a higher ratio of hypermethylated cfDNA DMRs to hypomethylated cfDNA DMRs (Visit A vs B) tended to demonstrate a faster time to clinical progression (TTP) ${ }^{55}$. Similarly, we examined the ENZR cell-line DMPs that were altered between: (1) $49 \mathrm{~F}^{\mathrm{ENZR}} / 42 \mathrm{D}^{\mathrm{ENZR}} / 42 \mathrm{~F}^{\mathrm{ENZR}}$ and $16 \mathrm{D}^{\mathrm{CRPC}}$ cells, (2) $49 \mathrm{~F}^{\mathrm{ENZR}}$ and $16 \mathrm{D}^{\mathrm{CRPC}}$ alone, and (3) $42 \mathrm{D}^{\mathrm{ENZR}} / 42 \mathrm{~F}^{\mathrm{ENZR}}$ and $16 \mathrm{D}^{\mathrm{CRPC}}$ cells alone. Firstly, DMPs that were hypermethylated in all ENZR cell lines $\left(49 \mathrm{~F}^{\mathrm{ENZR}} / 42 \mathrm{D}^{\mathrm{ENZR}} / 42 \mathrm{~F}^{\mathrm{ENZR}}\right)$ were overlaid with cfDNA DMRs (Visits A vs B). The ratio of these hypermethylated to hypomethylated DMRs (A vs B) was then correlated with TTP. There was a trend towards faster TTP for patients with increased cfDNA methylation at visit A for these regions $(\mathrm{P}=0.057)$ (Fig. 5a, b). This trend was not observed for hypermethylated DMPs only found in CRPC-Adeno/49F ${ }^{\text {ENZR }}$ cells (Figs. 5c, d). However, when we overlapped cfDNA DMRs with hypermethylated DMPs from the tNEPC cell lines $42 \mathrm{D}^{\mathrm{ENZR}} / 42 \mathrm{~F}^{\mathrm{ENZR}}$, there was a significant correlation with faster TTP (Figs. 5e, f). In order to assess whether this overlap between the array and cfDNA sequencing datasets was due to chance, we performed random sampling of the array dataset and compared the overlap of these probes with the cfDNA DMRs for each patient (Supplementary Table 25). There was a significant difference between the tNEPC-derived DMPs and randomly selected probes, suggesting that the tNEPC cell line derived methylation signals found within cfDNA samples is likely not due to random chance.

No correlation was observed for hypomethylated DMPs from any of these cell line comparisons (Supplementary Figure $7 \mathrm{a}-\mathrm{f}$ ). There was a slight correlation trend for DMPs hypermethylated in $42 \mathrm{D}^{\mathrm{ENZR}} / 42 \mathrm{~F}^{\mathrm{ENZR}} \mathrm{vs}^{\mathrm{N}} 4 \mathrm{~F}^{\mathrm{ENZR}}$ (Supplementary Figure $7 \mathrm{~g}$ ), but was not significant for hypomethylated DMPs (Supplementary Figure $7 \mathrm{~h}$ ). These findings suggest that $\mathrm{tNEPC}$ related methylation signals could serve as potential biomarkers. Some patients with faster TTP continued to exhibit high PSA levels in circulation ${ }^{55}$, which highlights disease heterogeneity, as these patients could harbor both CRPC-Adeno and CRPC-NE tumor cells.

\section{Discussion}

Overall, our analysis of the DNA methylome from the castration naïve state to treatment resistant CRPC suggests complex epigenetic changes in advanced PCa. We were able to track the changes in the methylome after successive treatments, including initial castration (representing conventional ADT) leading to CRPC state/cells followed by acquired resistance following enzalutamide treatment. Thus, mimicking progressive methylome changes as resistance emerges to various rounds of androgen-targeting treatment. In current clinical practice, the landscape of therapy sequences is complex, but still involving several types of androgen-targeting agents, chemotherapy, and for patients harboring CRPC-NE, potentially platinum-based therapies ${ }^{21}$. With each therapy, there are molecular alterations (genomic and epigenomic) that need to be tracked to determine optimal therapy sequences ${ }^{11,27}$. Therefore, it is important to distinguish the molecular alterations associated with androgen-pathway dependent disease, which could still be sensitive to androgen targeting agents and aberrations associated with tNEPC, which may benefit from other treatments. Furthermore, these molecular distinctions could help identify novel therapies for aggressive PCa. While this study was limited to a few representative cell lines, the advantage of this pre-clinical model was the ability to track the methylome at various stages of progression. Indeed, we observed methylation changes similar to those found in tumor tissue from tNEPC patients ${ }^{20}$.

In this study, we highlighted key methylome changes associated with the initial development of CRPC, then those following enzalutamide resistance and finally emergence of tNPEC-like disease. Despite sampling only a small fraction of the $\mathrm{CpG}$ sites in the genome, methylation alterations occurred in all genomic regions (i.e. promoters, gene bodies, and CGIs), and mostly within ENZR cells, especially in CRPC-NE/tNEPC like cells. While precise CpG sites may not have overlapped across all comparisons, several common genes and pathways were implicated within all ENZR cells, including those involved in developmental processes and hormone regulation, with more neurodevelopmental and morphogenic pathways enriched in tNEPC cells.

Interestingly, changes in the methylome appeared to have a potential impact on gene expression. While RNA data for CRPC-Adeno cells was not available, we noted similar methylation patterns in all ENZR cells, which was mostly associated with gene repression, including SLC25A43, which is deleted in certain breast tumors ${ }^{56}$, and a potential repressor of hypoxic response, ZFP36L1 $1^{57}$. Common ENZR upregulated genes involved in metastasis, $C O L 6 A 1^{48}$ and $A N T X R 1^{46}$, were also demonstrated. The genes implicated in all ENZR cells suggest potential targets beyond AR directed therapeutics that could impact growth and metastasis of both CRPC-Adeno and CRPC -NE cells, which requires further investigation. In addition, there were expression changes specific to tNEPC cells, including a known upregulated gene in CRPC-NE lesions, PEG10, which can regulate cell growth and invasion ${ }^{58}$. Other promoters of invasion/metastasis were also upregulated in tNEPC cells, such as FLOT2 $2^{51}$, $L A M B 1^{59}$, and GPR $19^{60}$. Although there was a slight increase in AR expression in ENZR/tNEPC cells, a previous study demonstrated siRNA knockdown of AR expression in these cells did not interfere with proliferation ${ }^{38}$. Furthermore, there are subsets of tumors with neuroendocrine features that also express AR, suggesting extensive plasticity in this disease state ${ }^{13,61}$. There were gene expression changes associated with low/altered AR activity, including reduced expression of TMPRSS2 and PSA/KLK3; however, many of these genes did not exhibit substantial methylation changes. This could be due to under-representation of CpGs near/within these genes 
a)

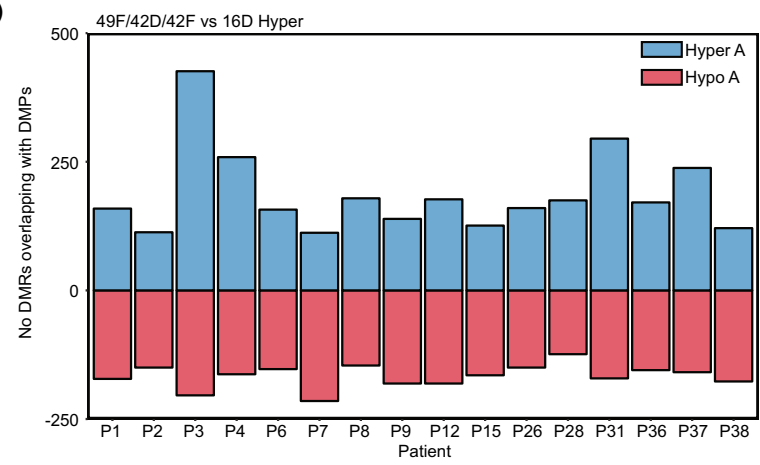

c)

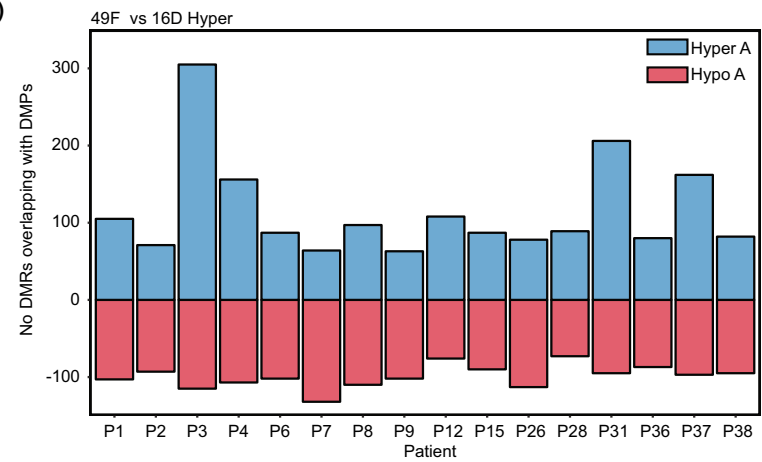

e)

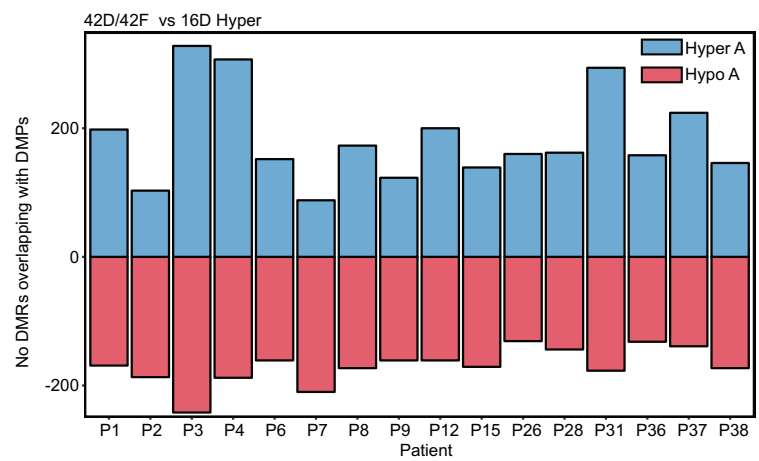

b)
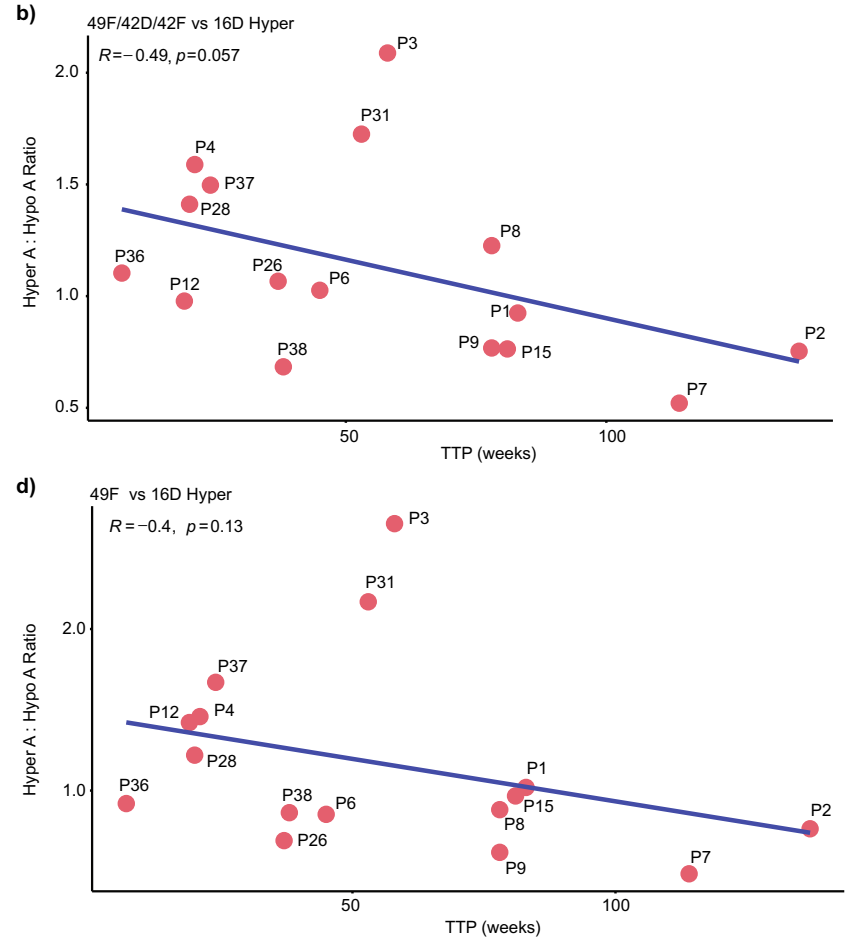

f)

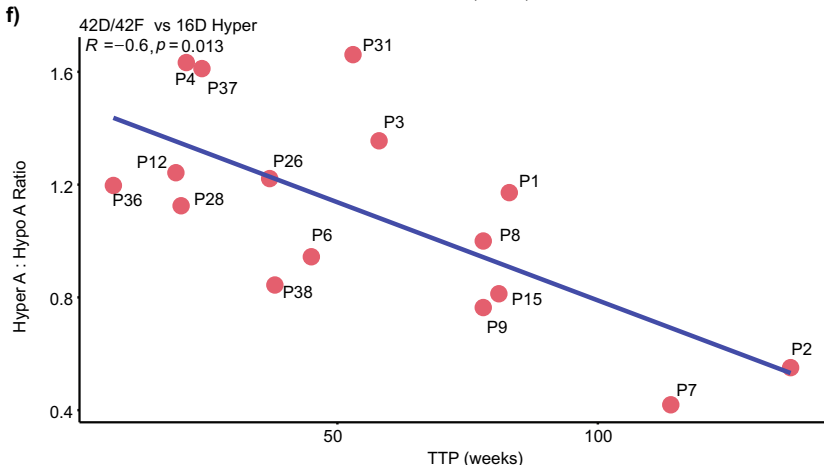

Figure 5. CRPC-NE related DMPs as potential biomarkers associated with clinical progression. The cell line DMPs that overlapped with cfDNA DMRs obtained from comparing the pre-treatment visit (visit A) and around 12-weeks during treatment (Visit B) were assessed. (a) The number of A vs B DMRs that overlapped with hypermethylated DMPs from comparing $49 \mathrm{~F}^{\mathrm{ENZR}} / 42 \mathrm{D}^{\mathrm{ENZR}} / 42 \mathrm{~F}^{\mathrm{ENZR}}$ vs $16 \mathrm{D}^{\mathrm{CRPC}}$ was quantified and separated by methylation trend for each patient (hypermethylated or hypomethylated in visit A vs B). (b) The ratio of these cfDNA DMRs (hypermethylated to hypomethylated) was correlated with TTP (Spearman rho and $\mathrm{p}$ value is shown). (c) Similarly, the number of DMRs that overlapped with $49 \mathrm{~F}^{\mathrm{ENZR}}$ vs $16 \mathrm{D}^{\mathrm{CRPC}}$ hypermethylated DMPs (not found in $42 \mathrm{D}^{\mathrm{ENZR}} / 42 \mathrm{~F}^{\mathrm{ENZR}}$ ) is shown in the barplot, and (d) correlation analysis with TTP is shown. (e) Finally, the number of cfDNA DMRs that contained hypermethylated DMPs from $42 \mathrm{D}^{\mathrm{ENZR}} / 42 \mathrm{~F}^{\mathrm{ENZR}} \mathrm{vs}$ $16 \mathrm{D}^{\mathrm{CRPC}}\left(\right.$ not $49 \mathrm{~F}^{\mathrm{ENZR}}$ ) was calculated, and (f) the ratio was also correlated with TTP.

in the array. Moreover, we observed that methylation changes in diverse pathways contributing to epigenomic instability in the tNEPC state.

We also assessed the potential of these methylation patterns as biomarkers of treatment resistance. By integrating the cell line methylation patterns with previously generated methylation profiles of cfDNA from mCRPC patients, we found potential markers of NE-like disease before patients initiated androgen-targeting agents. These markers were specific to the tNPEC cell lines and not CRPC-Adeno cells. In our prior study, extent of overlap between cfDNA DMRs and CRPC-NE tissue-derived signals also demonstrated a similar tendency towards faster clinical progression ${ }^{55}$. These combined findings highlight potential predictive biomarkers of resistance to androgen-targeting agents, requiring further validation in additional cohorts of patients.

\section{Methods}

CRPC cell line model. The CRPC cell line model was previously developed though serial transplantation of LNCaP-CRPC xenografts followed by castration and under the pressure of enzalutamide $(10 \mathrm{mg} / \mathrm{kg} / \mathrm{d})$ or vehicle control $^{24,25,38}$. This model was generated in accordance with approved animal use protocols in the Vancouver Prostate Centre, as specified by the Declaration of Helsinki for animal research. Cells were purified from xeno- 
grafted tumors representing various stages of PCa progression: pre-castration LNCaP tumor cells (LN), vehicle treated CRPC (V16D ${ }^{\mathrm{CRPC}}$ ) and ENZR cells $\left(42 \mathrm{D}^{\mathrm{ENZR}}, 42 \mathrm{~F}^{\mathrm{ENZR}}\right.$ and $\left.49 \mathrm{~F}^{\mathrm{ENZR}}\right)$. While all ENZR cell lines express $\mathrm{AR}, 49 \mathrm{~F}^{\mathrm{ENZR}}$ continues to secrete PSA and acquired an $A R$ activating mutation, whereas $42 \mathrm{D}^{\mathrm{ENZR}}$ and $42 \mathrm{~F}^{\mathrm{ENZR}}$ cells are PSA negative and do not possess this mutation ${ }^{38}$. Furthermore, $42 \mathrm{D}^{\mathrm{ENZR}} / 42 \mathrm{~F}^{\mathrm{ENZR}}$ express neuroendocrine lineage markers (i.e. chromagranin A and neural cell adhesion marker 1) as well as stem-like properties ${ }^{38}$.

DNA methylation and pathway analysis. DNA from each cell line was extracted using the QIAamp DNA mini Kit (Qiagen, Hilden, Germany) and quantified using Qubit dsDNA High Sensitivity Assay Kit (ThermoFisher Scientific, Waltham, MA, USA). We performed bisulfite conversion of $500 \mathrm{ng}$ of DNA for each sample (in triplicate) using the Zymo EZ DNA Methylation kit (Zymo, Irvine, CA, USA). This was followed by methylation analysis using the Infinium HumanMethylation450 BeadChip array using manufacturer's protocol (Illumina, San Diego, CA, USA). For each CpG site, beta value was calculated $[\beta=$ Methylated allele intensity $(\mathrm{M}) /($ Unmethylated allele intensity $(\mathrm{U})+$ Methylated allele intensity $(\mathrm{M})+100)]$. The Chip Analysis Methylation Pipeline (ChAMP) was used to analyze this dataset by first filtering out probes with low detection, SNP-related probes, and cross-reactive/multi-hit probes ${ }^{62}$. To adjust for probe type bias, normalization was performed using the BMIQ method ${ }^{63}$. Differentially methylated probes (DMPs) were then identified comparing each cell line with $\mathrm{FDR}<0.01$ and delta beta/log fold change $(\log F C) \geq 0.2$. All DMPs were annotated according to genomic location (i.e. TSS sites, genes). The champ.GSEA function was utilized to perform Gene Set Enrichment Analysis/Pathway analysis. Since the number of CpGs per gene varies, the gometh function was implemented to correct for this bias ${ }^{64}$.

Differential expression analysis. RNA expression data was previously published for LNCaP, $16 \mathrm{D}^{\mathrm{CRPC}}$, $42 \mathrm{D}^{\mathrm{ENZR}}$ and $42 \mathrm{~F}^{\mathrm{ENZR}}$ cell lines ${ }^{38}$. Alignments were performed using the STAR aligner and the hg19 genome build was used ${ }^{65}$. RPKM assignment and quantifications was done with CEMT RNA-seq pipeline using the Ensembl v75 gene models (http://www.epigenomes.ca/data/CEMT/methods/RNA-Seq.html). Differential expression analysis was performed using the DEfine algorithm ${ }^{66}$. Chi-squared $P$-value was estimated for every gene and Benjamini-Hochberg false discovery rate was applied (FDR $=0.05)$.

Additional statistical analysis. Unsupervised hierarchical clustering analysis of overall beta values was performed using the Ward clustering method in the base package of R (v3.5.3). Data was visualized using the VennDiagram package, the ggplots2 package, and heatmaps generated using the ComplexHeatmap package. Comparison with cfDNA-derived differentially methylated regions (DMRs) was performed using a published datase ${ }^{55}$. Briefly, cfDNA was collected from patients receiving enzalutamide or abiraterone acetate treatment at various timepoints, including baseline/prior to treatment initiation visit (A), around 12-weeks during treatment (visit B), and upon clinical progression (visit C). Genome-wide methylation sequencing analysis was performed followed by differential methylation analysis between study visits. Intra-patient DMRs were identified using the DMRHunter tool for each patient by comparing all visits ${ }^{55}$. The extent of overlap between cfDNA DMRs with ChAMP-derived DMPs from various cell line comparisons was calculated for each patient. The ratio of hypermethylated to hypomethylated DMRs (visit A vs B) was estimated and Spearman correlation analysis with time to clinical progression was assessed. To determine whether this overlap between datasets was due to random chance, we calculated the proportion of DMRs that overlapped with tNEPC DMPs as well as the percentage of these tNEPC DMPs that overlapped with the $450 \mathrm{~K}$ array probes for each patient (X\%). We then randomly sampled $(\mathrm{X} \%)$ probes $10,000 \times$ and determined the mean number of probes that overlapped with patient DMRs. We compared the proportion of random probes vs tNEPC probes within cfDNA DMRs using the one-proportion z-test.

All patients in this study provided informed written consent in accordance with approved institutional Research Ethics Board protocols from University Health Network (UHN) and Sinai Health System (SHS). Patients consented to the publication of study findings with unique study identifiers, which are not linked to personal health information. All work in this study was performed in accordance with the Declaration of Helsinki.

\section{Data availability}

All data analyzed during this study are included in this published article and in supplementary files.

Received: 20 October 2020; Accepted: 2 March 2021

Published online: 23 March 2021

\section{References}

1. Wong, Y. N., Ferraldeschi, R., Attard, G. \& de Bono, J. Evolution of androgen receptor targeted therapy for advanced prostate cancer. Nat. Rev. Clin. Oncol. 11, 365-376 (2014).

2. Sridhar, S. S. et al. Castration-resistant prostate cancer: from new pathophysiology to new treatment. Eur. Urol. 65, 289-299 (2014).

3. Crawford, E. D., Petrylak, D. \& Sartor, O. Navigating the evolving therapeutic landscape in advanced prostate cancer. Urol. Oncol. 35S, S1-S13 (2017).

4. Dai, C., Heemers, H. \& Sharifi, N. Androgen signaling in prostate cancer. Cold Spring Harb. Perspect. Med. 7, a030452 (2017).

5. Scher, H. I. et al. Increased survival with enzalutamide in prostate cancer after chemotherapy. N. Engl. J. Med. 367, 1187-1197 (2012).

6. Loriot, Y. et al. Effect of enzalutamide on health-related quality of life, pain, and skeletal-related events in asymptomatic and minimally symptomatic, chemotherapy-naive patients with metastatic castration-resistant prostate cancer (PREVAIL): results from a randomised, phase 3 trial. Lancet Oncol. 16, 509-521 (2015).

7. de Bono, J. S. et al. Abiraterone and increased survival in metastatic prostate cancer. N. Engl. J. Med. 364, 1995-2005 (2011). 
8. Ryan, C. J. et al. Abiraterone acetate plus prednisone versus placebo plus prednisone in chemotherapy-naive men with metastatic castration-resistant prostate cancer (COU-AA-302): final overall survival analysis of a randomised, double-blind, placebo-controlled phase 3 study. Lancet Oncol. 16, 152-160 (2015).

9. Saad, F. et al. 2019 Canadian Urological Association (CUA)-Canadian Uro Oncology Group (CUOG) guideline: management of castration-resistant prostate cancer (CRPC). Can. Urol. Assoc. J. 13, 307-314 (2019).

10. So, A. I. et al. Canadian Urological Association-Canadian Urologic Oncology Group guideline on metastatic castration-naive and castration-sensitive prostate cancer. Can. Urol. Assoc. J. 14, 17-23 (2020).

11. Armenia, J. et al. The long tail of oncogenic drivers in prostate cancer. Nat. Genet. 50, 645-651 (2018).

12. Bluemn, E. G. et al. Androgen receptor pathway-independent prostate cancer is sustained through FGF signaling. Cancer Cell 32, 474e476-489e476 (2017).

13. Aggarwal, R. et al. Clinical and genomic characterization of treatment-emergent small-cell neuroendocrine prostate cancer: a multi-institutional prospective study. J. Clin. Oncol. 36, 2492-2503 (2018).

14. Gonzalez-Billalabeitia, E., Conteduca, V., Wetterskog, D., Jayaram, A. \& Attard, G. Circulating tumor DNA in advanced prostate cancer: transitioning from discovery to a clinically implemented test. Prostate Cancer Prostatic Dis. 22, 195-205 (2019).

15. Robinson, D. et al. Integrative clinical genomics of advanced prostate cancer. Cell 162, 454 (2015).

16. Yegnasubramanian, S. Prostate cancer epigenetics and its clinical implications. Asian J. Androl. 18, 549-558 (2016).

17. Massie, C. E., Mills, I. G. \& Lynch, A. G. The importance of DNA methylation in prostate cancer development. J. Steroid Biochem. Mol. Biol. 166, 1-15 (2017).

18. Brocks, D. et al. Intratumor DNA methylation heterogeneity reflects clonal evolution in aggressive prostate cancer. Cell Rep. 8, 798-806 (2014).

19. Aryee, M. J. et al. DNA methylation alterations exhibit intraindividual stability and interindividual heterogeneity in prostate cancer metastases. Sci. Transl. Med. 5, 169 ra110 (2013).

20. Beltran, H. et al. Divergent clonal evolution of castration-resistant neuroendocrine prostate cancer. Nat. Med. 22, 298-305 (2016).

21. Puca, L., Vlachostergios, P. J. \& Beltran, H. Neuroendocrine Differentiation in Prostate Cancer: Emerging Biology, Models, and Therapies (Cold Spring Harb. Perspect, 2018).

22. Davies, A., Zoubeidi, A. \& Selth, L. A. The epigenetic and transcriptional landscape of neuroendocrine prostate cancer. Endocr. Relat. Cancer 27, R35-R50 (2020).

23. Dardenne, E. et al. N-Myc induces an EZH2-mediated transcriptional program driving neuroendocrine prostate cancer. Cancer Cell 30, 563-577 (2016).

24. Kuruma, H. et al. A novel antiandrogen, compound 30, suppresses castration-resistant and MDV3100-resistant prostate cancer growth in vitro and in vivo. Mol. Cancer Ther. 12, 567-576 (2013).

25. Matsumoto, H. et al. Cotargeting androgen receptor and clusterin delays castrate-resistant prostate cancer progression by inhibiting adaptive stress response and AR stability. Cancer Res. 73, 5206-5217 (2013).

26. Zelic, R. et al. Global DNA hypomethylation in prostate cancer development and progression: a systematic review. Prostate Cancer Prostatic Dis. 18, 1-12 (2015).

27. Zhao, S. G. et al. The DNA methylation landscape of advanced prostate cancer. Nat. Genet. 52, 778-789 (2020).

28. Hwang, J. H. et al. CREB5 promotes resistance to androgen-receptor antagonists and androgen deprivation in prostate cancer. Cell Rep. 29, 2355e2356-2370e2356 (2019).

29. Cancer Genome Atlas Research N. The molecular taxonomy of primary prostate cancer. Cell 163, 1011-1025 (2015).

30. Fanjul-Fernandez, M. et al. Cell-cell adhesion genes CTNNA2 and CTNNA3 are tumour suppressors frequently mutated in laryngeal carcinomas. Nat. Commun. 4, 2531 (2013).

31. Simovic, I., Castano-Rodriguez, N. \& Kaakoush, N. O. OPCML: a promising biomarker and therapeutic avenue. Trends Cancer 5, $463-466$ (2019).

32. Oksenberg, N. \& Ahituv, N. The role of AUTS2 in neurodevelopment and human evolution. Trends Genet. 29, 600-608 (2013).

33. Llamosas, N. et al. SYNGAP1 controls the maturation of dendrites, synaptic function, and network activity in developing human neurons. J. Neurosci. 40, 7980-7994 (2020).

34. Schayek, H. et al. Global methylation analysis identifies PITX2 as an upstream regulator of the androgen receptor and IGF-I receptor genes in prostate cancer. Horm. Metab. Res. 44, 511-519 (2012).

35. Gara, R. K. et al. Slit/robo pathway: a promising therapeutic target for cancer. Drug Discov. Today 20, 156-164 (2015).

36. Beltran, H. et al. Circulating tumor DNA profile recognizes transformation to castration-resistant neuroendocrine prostate cancer. J. Clin. Invest. 130, 1653-1668 (2020).

37. Baylin, S. B. \& Jones, P. A. Epigenetic determinants of cancer. Cold Spring Harb. Perspect. Biol. 8, a019505 (2016).

38. Bishop, J. L. et al. The master neural transcription factor BRN2 is an androgen receptor-suppressed driver of neuroendocrine differentiation in prostate cancer. Cancer Discov. 7, 54-71 (2017).

39. Sharma, N. L. et al. The androgen receptor induces a distinct transcriptional program in castration-resistant prostate cancer in man. Cancer Cell 23, 35-47 (2013).

40. Lee, G. T. et al. WNT5A induces castration-resistant prostate cancer via CCL2 and tumour-infiltrating macrophages. Br. J. Cancer 118, 670-678 (2018).

41. Yamamoto, H. et al. Wnt5a signaling is involved in the aggressiveness of prostate cancer and expression of metalloproteinase. Oncogene 29, 2036-2046 (2010).

42. Sharma, A. et al. The prostate metastasis suppressor gene NDRG1 differentially regulates cell motility and invasion. Mol. Oncol. 11, 655-669 (2017).

43. Hsu, C. M. et al. Upregulated SLC22A3 has a potential for improving survival of patients with head and neck squamous cell carcinoma receiving cisplatin treatment. Oncotarget 8, 74348-74358 (2017).

44. Leon, K. et al. Structural basis for adhesion G protein-coupled receptor Gpr126 function. Nat. Commun. 11, 194 (2020).

45. Wang, W. Y. et al. Stimulative role of ST6GALNAC1 in proliferation, migration and invasion of ovarian cancer stem cells via the Akt signaling pathway. Cancer Cell Int. 19, 86 (2019).

46. Chen, D., Bhat-Nakshatri, P., Goswami, C., Badve, S. \& Nakshatri, H. ANTXR1, a stem cell-enriched functional biomarker, connects collagen signaling to cancer stem-like cells and metastasis in breast cancer. Cancer Res. 73, 5821-5833 (2013).

47. Fan, L. et al. CXCL13 is androgen-responsive and involved in androgen induced prostate cancer cell migration and invasion. Oncotarget 8, 53244-53261 (2017).

48. Owusu-Ansah, K. G. et al. COL6A1 promotes metastasis and predicts poor prognosis in patients with pancreatic cancer. Int. J. Oncol. 55, 391-404 (2019).

49. Spanjol, J. et al. Role of bone morphogenetic proteins in human prostate cancer pathogenesis and development of bone metastases: immunohistochemical study. Coll. Antropol. 34(Suppl 2), 119-125 (2010).

50. Ganguly, S. S. et al. Notch3 promotes prostate cancer-induced bone lesion development via MMP-3. Oncogene 39, 204-218 (2020).

51. Wang, C. H. et al. Flot2 promotes tumor growth and metastasis through modulating cell cycle and inducing epithelial-mesenchymal transition of hepatocellular carcinoma. Am. J. Cancer Res. 7, 1068-1083 (2017).

52. Yin, X. et al. Protocadherin 17 functions as a tumor suppressor suppressing Wnt/beta-catenin signaling and cell metastasis and is frequently methylated in breast cancer. Oncotarget 7, 51720-51732 (2016).

53. Kim, S. et al. PEG10 is associated with treatment-induced neuroendocrine prostate cancer. J. Mol. Endocrinol. 63, 39-49 (2019). 
54. Zhang, P., Schaefer-Klein, J., Cheville, J. C., Vasmatzis, G. \& Kovtun, I. V. Frequently rearranged and overexpressed delta-catenin is responsible for low sensitivity of prostate cancer cells to androgen receptor and beta-catenin antagonists. Oncotarget 9, 24428-24442 (2018).

55. Peter, M. R. et al. Dynamics of the cell-free DNA methylome of metastatic prostate cancer during androgen-targeting treatment. Epigenomics 12, 1317-1332 (2020).

56. Tina, E. et al. The mitochondrial transporter SLC25A43 is frequently deleted and may influence cell proliferation in HER2-positive breast tumors. BMC Cancer 12, 350 (2012).

57. Loh, X. Y. et al. RNA-binding protein ZFP36L1 suppresses hypoxia and cell-cycle signaling. Cancer Res. 80, 219-233 (2020).

58. Akamatsu, S. et al. The placental gene PEG10 promotes progression of neuroendocrine prostate cancer. Cell Rep. 12, 922-936 (2015).

59. Govaere, O. et al. The PDGFRalpha-laminin B1-keratin 19 cascade drives tumor progression at the invasive front of human hepatocellular carcinoma. Oncogene 36, 6605-6616 (2017).

60. Rao, A. \& Herr, D. R. G protein-coupled receptor GPR19 regulates E-cadherin expression and invasion of breast cancer cells. Biochim. Biophys. Acta Mol. Cell Res. 1864, 1318-1327 (2017).

61. Labrecque, M. P. et al. Molecular profiling stratifies diverse phenotypes of treatment-refractory metastatic castration-resistant prostate cancer. J. Clin. Invest. 129, 4492-4505 (2019)

62. Tian, Y. et al. ChAMP: updated methylation analysis pipeline for Illumina BeadChips. Bioinformatics 33, 3982-3984 (2017).

63. Teschendorff, A. E. et al. A beta-mixture quantile normalization method for correcting probe design bias in Illumina Infinium 450 k DNA methylation data. Bioinformatics 29, 189-196 (2013).

64. Phipson, B., Maksimovic, J. \& Oshlack, A. missMethyl: an R package for analyzing data from Illumina's HumanMethylation450 platform. Bioinformatics 32, 286-288 (2016).

65. Dobin, A. et al. STAR: ultrafast universal RNA-seq aligner. Bioinformatics 29, 15-21 (2013).

66. Savic, N. et al. Histone chaperone paralogs have redundant, cooperative, and divergent functions in yeast. Genetics 213, $1301-1316$ (2019).

\section{Acknowledgements}

Methylation array profiling and initial data processing was performed by The Centre for Applied Genomics (TCAG, Hospital for Sick Children, Toronto, Canada). The UHN Genitourinary clinic and Genitourinary Biobank recruited patients, collected blood samples and provided patient follow-up data.

\section{Author contributions}

B.B. and A.Z. designed the overall study. A.Z. developed the xenograft model and A.D. isolated DNA from cultured cell lines. M.R.P. performed sample processing for the methylation array, differential methylation analysis, as well as integration with gene expression and cfDNA methylation datasets. M.B. developed the DEfine pipeline and performed differential expression analysis, and M.H. contributed to cfDNA analysis. R.I. and G.D.B. assisted with pathway analysis. A.D. and N.E.F. were also involved in the data analysis and interpretation. M.R.P., M.B., A.D., A.Z. and B.B. composed the manuscript with feedback from all co-authors involved.

\section{Funding}

This study was supported by a Prostate Cancer Canada Movember Discovery Grant (D2014-10) and an Astellas Prostate Cancer Innovation Fund (2017) to B. Bapat.

\section{Competing interests}

The authors declare no competing interests.

\section{Additional information}

Supplementary Information The online version contains supplementary material available at https://doi.org/ 10.1038/s41598-021-85812-3.

Correspondence and requests for materials should be addressed to B.B.

Reprints and permissions information is available at www.nature.com/reprints.

Publisher's note Springer Nature remains neutral with regard to jurisdictional claims in published maps and institutional affiliations.

(c) (i) Open Access This article is licensed under a Creative Commons Attribution 4.0 International License, which permits use, sharing, adaptation, distribution and reproduction in any medium or format, as long as you give appropriate credit to the original author(s) and the source, provide a link to the Creative Commons licence, and indicate if changes were made. The images or other third party material in this article are included in the article's Creative Commons licence, unless indicated otherwise in a credit line to the material. If material is not included in the article's Creative Commons licence and your intended use is not permitted by statutory regulation or exceeds the permitted use, you will need to obtain permission directly from the copyright holder. To view a copy of this licence, visit http://creativecommons.org/licenses/by/4.0/.

(C) The Author(s) 2021 